\title{
Thermal properties of AIN-based atom chips
}

\author{
J. Armijo, C. L. Garrido Alzar and I. Bouchoule \\ Laboratoire Charles Fabry de l'Institut d'Optique, UMR 8501 du CNRS, 91127 Palaiseau Cedex, France
}

\begin{abstract}
We have studied the thermal properties of atom chips consisting of high thermal conductivity Aluminum Nitride (AlN) substrates on which gold microwires are directly deposited. We have measured the heating of wires of several widths and with different thermal couplings to the copper mount holding the chip. The results are in good agreement with a theoretical model where the copper mount is treated as a heat sink and the thermal interface resistance between the wire and the substrate is vanishing. We give analytical formulas describing the different transient heating regimes and the steady state. We identify criteria to optimize the design of a chip as well as the maximal currents $I_{c}$ that can be fed in the wires. For a $600 \mu \mathrm{m}$ thick-chip glued on a copper block with Epotek H77, we find $I_{c}=16$ A for a $3 \mu \mathrm{m}$ high, $200 \mu \mathrm{m}$ wide-wire.

PACS numbers: $39.25 .+\mathrm{k}, 03.75 . \mathrm{Be}$
\end{abstract}

\section{INTRODUCTION}

A few years after the first Bose Einstein condensates (BEC) in atomic vapours, the will to miniaturize the set-ups has led to the production of atom chips, which consist in microfabricated elements, most usually wires, that are used to trap and manipulate cold atomic clouds. The atom chip soon became increasingly popular as a compact, robust and versatile device suitable for the production of BEC [1-3] and for studies on quantum matter, cold atom-based metrology, or quantum information [4].

The major interest of atom chips is to manipulate atoms in the close vicinity of the field sources, so that trapping potentials with strong spatial variations can be obtained at very low power consumption. Small structures and tightly confining traps allow one to perform efficient evaporative cooling at high collision rates, to squeeze cold clouds to very anisotropic geometries so that low dimensional regimes are reached [58], and make it possible to realize a great diversity of trapping geometries [4].

Still, there are limits to the miniaturization of the structures and for several reasons, large currents can still be needed. First, as the loading stages require traps deep and wide enough to collect a high number of atoms [9], big structures running large currents are needed. These large loading structures can be placed below the chip [10], but other limitations still prevent the use of arbitrarily small structures. One problem is that when atoms are brought close to the source wire they become sensitive to the potential roughness created by the wire's imperfections [11, 12]. Second, it is often desirable to avoid the interactions between the atoms and the surface (Casimir-Polder force [13, 14] or Johnson noise causing spinflip losses [15, 16]), which involves keeping the atoms at least some microns away from it. For all those reasons, the maximal current that can be carried by each wire is a crucial parameter determining the possibilities of an atom chip. Unless one uses superconducting wires [17], in which case the maximal current is the critical current above which the metal becomes normal, the maximal current is determined by the dissipation of the heat generated in the wires. In all this paper, we only consider resistive wires, much simpler to achieve experimentally and most widely used.
Up to now, atom chips have mainly been realized on Silicon $(\mathrm{Si})$ wafers because $\mathrm{Si}$ is cheap, fabrication techniques are well developed, and it is a good thermal conductor. But, as $\mathrm{Si}$ is semiconductor, an electrically insulating layer, generally $\mathrm{SiO}_{2}$, needs to be placed between the wafer and the metallic wires. Unfortunately, $\mathrm{SiO}_{2}$ is also a thermal insulator, and, in a previous study [18], it was found that this layer is the main limitation to the removal of heat in Si-based atom chips. On the other hand, AlN is a substrate material that has been especially selected for being simultaneously a good electrical insulator and a good thermal conductor. It was first used for high-power microelectronics applications [19]. More and more groups working on atom chips are now moving to AlN substrates [9, 20-22], because they allow for direct deposition of the wires on the substrate. Since no thermal contact resistance between the wire and the substrate is expected, much better heat dissipation is foreseen.

We have fabricated such chips and measured their thermal behavior. We first present the model that we have developed to understand our experiments and identify the different heating regimes. We then show that this model reproduces very well the measured wire heating for different wire widths, different thermal couplings to the copper mount and within the different heating regimes. In particular, we show that the heating in AlN-based atom chips is only governed by heat diffusion in the substrate, unlike the Si-based atom chips. Therefore, the relevant phenomenon to consider for the current limitations is the long-time heating rather than a fast heating due to a thermal resistance between the wire and the substrate. The thermal coupling to the copper mount and the possibility to quickly reach a stationary regime thus acquire a crucial importance. Finally, we draw pratical conclusions on the optimal design and the current limitations of AlN-based atom chips.

\section{THEORETICAL MODEL}

At any time of its operation, the temperature of a currentcarrying wire is the result of its resistive heating and the heat removal via different channels. Most of the heat is removed by conduction through the substrate on which the wire is deposited, out to the chip mount, considered as a heat reservoir. When the chip is placed in a vacuum chamber, the only other 


\begin{tabular}{|l|l|l|}
\hline$\lambda$ & AlN conductivity & $128 \mathrm{~W} /(\mathrm{Km})$ \\
$D$ & $\lambda / c:$ AlN diffusion constant & $53 \mathrm{~mm}^{2} / \mathrm{s}$ \\
$\sigma$ & thermal contact resistance between & $1.1 \times 10^{-4} \mathrm{~m}^{2} \mathrm{~K} / \mathrm{W}$ \\
& substrate and heat sink & \\
$e$ & substrate thickness & $600 \mu \mathrm{m}$ \\
$l_{c}$ & $\lambda \sigma:$ contact length & $14 \mathrm{~mm}$ \\
$l_{s}$ & $\sqrt{e l_{c}}:$ stationary length & $2.9 \mathrm{~mm}$ \\
$t_{s}$ & $l_{s}^{2} / D=\sigma e c:$ stationary time \\
$t_{e}$ & $e^{2} / D:$ crossover time between $2 D$ & $0.16 \mathrm{~s}$ \\
& and $1 D x$ regimes & $6.7 \mathrm{~ms}$ \\
$\Phi$ & resistive power generated by the wire \\
per unit length & $920 \mathrm{Wm}^{-1}$ \\
\hline
\end{tabular}

TABLE I: Relevant parameters for the wire heating and their value in the case experimentally studied in this paper where the chip is glued onto the copper block. The value of $\Phi$ is given for a $200 \mu \mathrm{m}$ wide, $3 \mu \mathrm{m}$ high gold wire running a current of $5 \mathrm{~A}$.

mechanism for heat dissipation is black-body radiation of the wire which, as we show later, has a negligible effect. In air, as for some of the experiments presented below, air convection can also play a role, but it is expected to be negligible, in particular since the wires in the chip we use are covered by a $6 \mu \mathrm{m}$ thick-layer of resist of high thermal resistance. In the following, we thus assume that heat conduction inside the substrate is the only mechanism for heat removal and we compute the expected heating of the wire.

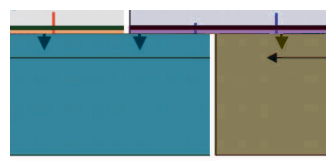

FIG. 1: Sketch of our model. The substrate is infinite in the $x$ direction and the $\mathrm{Cu}$ mount acts as a heat reservoir. The heat dissipated in the wire diffuses in the substrate in the $1 \mathrm{Dz}, 2 \mathrm{D}$ and $1 \mathrm{Dx}$ regimes successively. When the transverse spread is $l_{s}$, an equilibrium state is reached (see text). (color online)

In our model, depicted in Fig. 1, we assume that the wire, of width $W$ along the $x$ direction is infinite in the $y$ direction. We also assume that the substrate of thickness $e$ is infinite in the transverse direction $\mathrm{x}$. The back surface of the substrate is supposed to be in contact with a heat reservoir at temperature $T_{0}$ with a thermal contact resistance $\sigma$ (in $\mathrm{m}^{2} \mathrm{~K} / \mathrm{W}$ ). Finally, we assume that the energy flux per unit area from the wire to the substrate is homogeneous over the wire width. Using these hypothesis, we compute the temperature inside the substrate and in particular just below the wire. We assume that the thermal contact resistance between the wire and the substrate is vanishing so that the substrate temperature at the wire position is equal to the wire temperature.

The substrate thermal properties are described by its specific heat per unit volume $c$ and its thermal conductivity $\lambda$. The resulting diffusion constant is $D=\lambda / c$. With the thermal contact resistance between the substrate and the heat reservoir, we can construct a length $l_{c}=\lambda \sigma$. However, a more relevant length scale is $l_{s}=\sqrt{e l_{c}}$, denoted as the stationary length, as we explain in the following. Thus, three different length scales govern the heat diffusion process : $W, e$ and $l_{s}$. From these lengths we derive the corresponding time scales $t_{W}=W^{2} / D, t_{e}=e^{2} / D$ and $t_{s}=l_{s}^{2} / D$. Table \gathers the most relevant parameters and gives their value for the experimental case studied in this paper, in the most favorable situation where the chip is glued onto the copper block.

Let us first consider the step response of the system for a wire of constant resistivity : the wire and substrate are at temperature $T_{0}$ when the current is suddenly turned on, producing a heat flow from the wire to the substrate per unit length and per unit time $\Phi$. We compute the evolution of the wire temperature, noting by $\Delta T$ its deviation from $T_{0}$. In the equations, we express temperature in energy unit, setting $k_{B}=1$. To give some physical insight we consider the situation where the characteristic times satisfy $t_{W} \ll t_{e} \ll t_{s}$, a situation usually fulfilled. For times $t \ll t_{W}$, the energy has diffused inside the substrate over lengths much smaller than the wire width $W$. Then the diffusion inside the substrate is expected to be one-dimensional in the $\mathrm{z}$ direction (see Fig.11) and in this $1 D z$ regime the wire heating can be written as

$$
\Delta T^{1 D z}=2 \frac{\Phi}{W} \frac{1}{\sqrt{\pi c \lambda}} \sqrt{t}
$$

Next, for times $t_{W} \ll t \ll t_{e}$, the energy has diffused into the substrate over lengths much larger than the wire width but much smaller than the substrate thickness $e$, so that the heat diffusion is well described by a two-dimensional model (see Fig. 10 and we expect the temperature to increase as

$$
\Delta T^{2 D}=\frac{\Phi}{2 \pi \lambda} \ln \left(\frac{W_{\mathrm{eff}}^{2}+2 D t}{W_{\mathrm{eff}}^{2}}\right)
$$

where $W_{\text {eff }}$ is an effective width on the order of $W$. At times $t \gg t_{e}$, the energy has diffused over lengths much larger than the substrate thickness $e$, so one expects now a onedimensional model in the $\mathrm{x}$ direction (see Fig. 1) to be an accurate description of the wire heating, which takes the form

$$
\Delta T^{1 D x}=\Phi \frac{1}{e \sqrt{\pi c \lambda}} \sqrt{t} .
$$

This formula is valid as long as the energy flux per unit length to the reservoir $\Phi_{R}$ is much smaller than $\Phi$. The heat flux to the reservoir per unit area is $j_{R}=\left(T(x)-T_{0}\right) / \sigma$ where $T(x)$ is the substrate temperature at position $x$. Using $T(0)-T_{0}=$ $\Delta T$ and since $T(x)-T_{0}$ falls off to zero over a width of order 


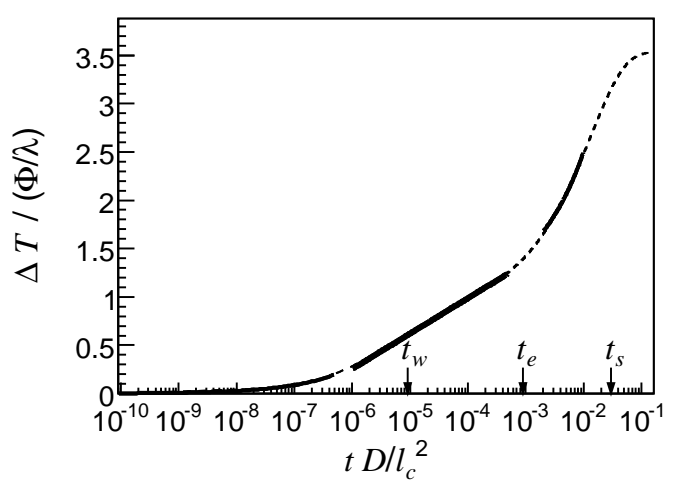

FIG. 2: Transient wire heating for $W=3 \times 10^{-3} l_{c}$ and $e=3 \times$ $10^{-2} l_{c}$ (dashed line). Temperature and time are scaled to $\Phi / \lambda$ and $t_{c}=l_{c}^{2} / D$ respectively. The solid lines correspond to fits with the functions (from earlier to later times) $a_{1} * \Delta T^{1 D z}, \Delta T^{2 D}$ and $a_{3}+$ $b_{3} \Delta T^{1 D x}$, the fitted parameters being $a_{1}=0.74, W_{\text {eff }}=0.17 W$, $a_{3}=1.0$ and $b_{3}=0.79$.

$L=\sqrt{D t}$, we find that $\Phi_{r}=\int d x j_{R} \simeq \Delta T \sqrt{D t} / \sigma$. Inserting in Eq. 3, we find $\Phi_{R} \simeq t \Phi D /\left(e l_{c}\right)=\Phi t / t_{s}$. Thus Eq. 3 is valid as long as $t \ll t_{s}$. For $t \simeq t_{s}, \Phi_{R} \simeq \Phi$ : the heat flux to the reservoir compensates the input heat flux $\Phi$ and the system reaches a steady state. This justifies the denotation of $t_{s}$ and $l_{s}$ as the stationary time and length respectively (see Fig. 11. Interestingly, $t_{s}$, which can be rewritten as $t_{s}=c e \sigma$, does not depend on $\lambda$. Note also that $t_{s}$ is equal to the relaxation time of the substrate, defined as the time constant of the exponential decay of the substrate temperature after a homogeneous heating. This can be understood by the intuitive picture that the heat spreads in the substrate as long as it has not been absorbed by the mount.

When the stationary flow is finally established, the equilibrium temperature at the wire can be estimated by summing up the contributions of the three successive regimes. The contribution of the late $1 D x$ regime is found replacing $t=t_{s}$ in Eq. 3. $T_{e q}^{1 D x}=\alpha \frac{\Phi}{\lambda} \sqrt{\frac{l_{c}}{e}}$, where $\alpha$ is a numerical prefactor. From Eq. 2 we find that the $2 D$ regime contributes to an amount $T_{e q}^{2 D}=\frac{\Phi}{\pi \lambda} \ln \left(\frac{e}{W_{0}}\right)$ where $W_{0}$ is of the order of W. As for the initial $1 D z$ regime, its contribution scales as $\frac{\Phi}{\lambda}$ so that it is possible to incorporate it in $T_{e q}^{2 D}$ by renormalizing $W_{0}$. Finally we get the analytic expression for the equilibrium temperature

$$
\Delta T_{e q}=\frac{\Phi}{2 \lambda} \sqrt{\frac{l_{c}}{e}}+\frac{\Phi}{\pi \lambda} \ln \left(\frac{e}{W_{0}}\right)
$$

where we have replaced $\alpha$ by $\frac{1}{2}$ a posteriori from the calculation that we explain below.

In order to get quantitative predictions and to investigate the crossovers between the different diffusion regimes, it is useful to perform numerical calculations. For this purpose we use standard Fourier analysis. For an input heat flow at the top of the substrate that varies as $j_{k, \omega} e^{i k x} e^{i \omega t}$, we find the temperature at $x, z=0$

$$
\Delta T_{k, \omega}=j_{k, \omega} \frac{\operatorname{Sinh}(\mathrm{Ke}) / l_{\mathrm{c}}+\mathrm{KCosh}(\mathrm{Ke})}{\operatorname{Cosh}(\mathrm{Ke}) / \mathrm{l}_{\mathrm{c}}+\mathrm{KSinh}(\mathrm{Ke})} \frac{1}{K \lambda},
$$

where $K=\sqrt{k^{2}+i c \lambda \omega}$. We make the assumption that the heat flow $\mathrm{j}(\mathrm{x})$ is homogeneous over the wire size. The equilibrium temperature is then obtained setting $\omega$ to 0 in Eq. 5 . In the case $l_{c} \gg e \gg W$, the wavevectors much smaller than $1 / e$ give a lorentzian contribution, whose integral yields the prefactor $\alpha=1 / 2$ for the first term in rhs of Eq. 4.

Most importantly, we use Eq. 5 to compute the transient heating of the wire. Fig. 2 shows the result of the calculation for an input energy flux $\Phi$ which is a step function in time, for well separated length scales. Fits in the three different temporal domains are in good agreement with the analytical laws derived above. We observe that the initial $1 D z$ behavior fails noticeably already for $t \simeq t_{W} / 10$ and that $W_{\text {eff }}$ in Eq. 2 is noticeably smaller than $W\left(W_{\text {eff }} \simeq 0.17(3) W\right)$. On the other hand, the transition from the $2 D$ to the $1 D x$ regime and the convergence to the equilibrium state occur as expected for $t \simeq t_{e}$ and $t \simeq t_{s}$ respectively.

The above calculations assume that the energy flow $\Phi$ is constant in time, however, as the wire heats up, its resistivity, and consequently $\Phi$ as well, also increase. To take this effect into account numerically, we compute the wire temperature as

$$
T(t)=T_{0}+\int_{0}^{t} R\left(t-t^{\prime}\right) \Phi\left(t^{\prime}\right) d t^{\prime}
$$

where the impulse response function $R\left(t^{\prime}\right)$ is determined using Eq. 5. To compute $\Phi\left(t^{\prime}\right)$ at a given $t^{\prime}$, we assume a homogeneous wire temperature, so that $\Phi\left(t^{\prime}\right)=I^{2} \rho\left(T\left(t^{\prime}\right)\right) / h W$. This approximation is justified a posteriori since the computed wire temperature inhomogeneity never exceeds $10 \%$ for the parameters explored in this paper. The dependence of the gold resistivity with temperature is approximated by

$$
\rho=\rho_{0}(1+\alpha \Delta T)
$$

where $\alpha=0.0038 \mathrm{~K}^{-1}$ is obtained from a linear fit of the reported values of $\rho$ between $200 \mathrm{~K}$ and $500 \mathrm{~K}$ [23].

Our last remark about the model concerns the assumption that the input energy flux $j(x)$ is homogeneous over the wire width. This is expected to be true if there is no spatial redistribution of the energy released by Joule's effect inside the wire, but might be inaccurate if the wire height is not small compared to its width or if the wire thermal diffusion constant is very large compared to that of the substrate. In the extreme case where the wire temperature is uniform, simple calculations can be done, to provide an upper bound on the error. More precisely, for $e \gg W$ and for times much larger than $t_{W}$, we find[25] $j(x) \simeq 2 \Phi /\left(\pi W \sqrt{1-(2 x / W)^{2}}\right)$. Using Eq. 5, we then find that the equilibrium temperature is lower than in the case of a uniform $j(x)$ by an amount smaller than $5 \%$ for all the parameters explored in this article. Thus, we expect that the error in our predictions caused by our assumption on $j(x)$ cannot be larger than 5\%. Alternatively, the small wire temperature inhomogeneity predicted by our model shows that both boundary conditions give similar results. 


\section{COMPARISON WITH MEASUREMENTS}

We now come to the experimental measurements and their comparison with the model. The chip is based on an AlN substrate of thickness $600(50) \mu \mathrm{m}$ and of size $25 \mathrm{~mm} \times 35 \mathrm{~mm}$. Gold wires of height $3 \mu \mathrm{m}$ are deposited by evaporation on the substrate, on top of a $30 \mathrm{~nm}$ thick-titanium adhesion layer. We measure the wire heating after a constant current is turned on by monitoring the wire resistance and using Eq.7 The current supplies we use allow a current rise time of about $10 \mu \mathrm{s}$. The wire resistance is deduced from the voltage drop across it. The contribution $R_{c}$ of the connecting wires and the contact resistances has to be subtracted from the measured resistance. To compute $R_{c}$, we measure the circuit resistance at a current low enough to produce negligible heating and subtract the contribution of the microwire, computed using the nominal wire dimensions and the gold resistivity value at $300 \mathrm{~K}$. We perform heating measurements on two different wires : a $200 \mu \mathrm{m}$ wide, $20 \mathrm{~mm}$ long-wire and a $7 \mu \mathrm{m}$ wide, $3 \mathrm{~mm}$ long-wire.

To investigate the heating on long time scales we use the $200 \mu \mathrm{m}$ wide-wire, in which we run a current of about $5 \mathrm{~A}$. Three main cases are considered. First, the chip is laid on paper to thermally insulate it from the copper block. Second, it is maintained on the copper block, either in air or in vacuum. Finally, the chip is glued on the copper block with Epotek H77.

In the case where the substrate is laid on paper, we compare the data to the theoretical model, assuming that no heat escapes the substrate $\left(l_{c} \rightarrow \infty\right)$. As shown in Fig. III we find agreement within a few percent for times lower than $2 \mathrm{~s}$, provided the AlN thermal conductivity is set to $\lambda=128 \mathrm{~W} /(\mathrm{Km})$. This value is close to the reported values for AlN [19] although somewhat smaller. The discrepancy may compensate for imprecisions on the other parameters (AlN heat capacity, gold resistivity, wire size and substrate thickness) which are fixed to their nominal values. In all the following, we use this fitted value of $\lambda$. With our substrate $t_{e} \simeq 7 \mathrm{~ms}$, so that for the times considered in Fig. III, one expects the heating process to be well explained by the $1 D x$ model of Eq. 3. Using this simple model and including the dependence of gold resistivity with temperature, we obtain the short-dashed curve shown in Fig. III] It agrees within 5\% with the more complete calculation. The discrepancy, which is about a constant offset, is due to the early $2 D$ regime. At times longer than $2.5 \mathrm{~s}$, the data show an excess heating compared to the model. We attribute this effect to the finite size of the substrate : after $t=2 \mathrm{~s}$ the heat has diffused over a typical distance $\sqrt{D t}=1.1 \mathrm{~cm}$, larger than the distance from the wire to the substrate edge $(1.0 \mathrm{~cm})$.

In the case where the substrate is maintained on copper, the relevant new parameter is the thermal contact resistance $\sigma$ between the substrate and the copper block. We have measured $\sigma$ in the following way. First, we heat the substrate to a temperature $T_{1} \simeq 500 \mathrm{~K}$ by running current in a wire and letting the substrate thermalize for a few seconds, while keeping it isolated from the copper with some paper. Then, we suddenly remove the paper so that the substrate is in thermal contact with the copper block, with an applied pressure of $2 \times 10^{3} \mathrm{~Pa}$. We monitor the thermal relaxation of the substrate by recording the resistance of one of the chip wires in which a small current of $10 \mathrm{~mA}$ is constantly flowing. A fit to the function $T=T_{0}+\left(T_{1}-T_{0}\right) \exp (-t / \tau)$ yields the relaxation time $\tau=\sigma c e=0.82 \mathrm{~s}$, which gives the thermal contact resistance $\sigma=5.77 \times 10^{-4} \mathrm{~m}^{2} \mathrm{~K} / \mathrm{W}$. To our knowledge no measurement of $\sigma$ was previoulsy reported for such a small contact pressure at a $\mathrm{Cu}-\mathrm{AlN}$ interface. A much better coupling could be obtained for pressures higher than $10^{5} \mathrm{~Pa}$ [24], but such high pressures do not seem realistic in the context of atom chips, because usually a large free surface on the chip is required. The value of $\sigma$ also depends on the surface quality, so we need to mention that the substrate we use has a backside roughness amplitude of about $100 \mathrm{~nm}$. Importing in the model the measured value of $\sigma$, we compute the heating of the wire after a 5 A current-step, with no adjustable parameters. The result, shown as a dotted curve in Fig. III agrees with the measurement to better than $10 \%$, which is a good validation for our model.

In real experiments, unless the chip is itself one of the walls of the vacuum chamber [22], the contact surface between the chip and the heat sink is in vacuum. We have thus also studied the case of a chip maintained on a copper block in vacuum, at a pressure lower than $10^{-1}$ mbar. We find that the thermal contact resistance $\sigma_{\text {vac }}$ between the substrate and the copper is much larger than in air, meaning that the thermal coupling in room conditions is actually caused by the air present in the voids between the substrate and the copper block. To measure $\sigma_{\text {vac }}$, we monitor the cooling of the substrate with the same protocol as in air, with the difference that, since the cooling time is larger than the thermalization time of the substrate (a few seconds), thermal insulation of the substrate in the initial heating stage is not needed. The measured relaxation time is $\tau_{\text {vac }}=26 \mathrm{~s}$, corresponding to $\sigma_{\mathrm{vac}}=1.8 \times 10^{-2} \mathrm{~m}^{2} \mathrm{~K} / \mathrm{W}$. We also measure the relaxation time in vaccuum for a chip laid on paper and find $82 \mathrm{~s}$, a value several times larger than $\tau_{\text {vac. }}$. This confirms that conduction to the copper is the main cooling mechanism for a chip held on copper, even in vacuum where the thermal resistance $\sigma_{\text {vac }}$ is high, and that black-body radiation is negligible. In practice, since the stationary time $t_{s}$ is identical to the relaxation time $\tau$, the coupling to the copper block has negligible effect on the wire heating for times much smaller than $\tau$. In particular, within the time of our measurement (4s), we observed a heating equal within $5 \%$ to the one observed when the chip is laid on paper.

In order to mitigate the wire heating, especially if operating in vacuum, the previous studies show that it is highly desirable to fill the voids between the chip and its mount. That is why, in the last situation, we have glued the chip onto the copper block with a thin layer of Epotek H77, a glue of high heat conductivity (according to the specifications, $\lambda_{H 77}=0.66 \mathrm{~W} /(\mathrm{mK})$ ) containing grains whose maximal radius is specified to 20 $\mu m$. The experimental value of the steady state temperature (see Fig. III) is obtained, within our model, for a thermal contact resistance $\sigma=1.1 \times 10^{-4} \mathrm{~m}^{2} \mathrm{~K} / \mathrm{W}$, which corresponds to a glue layer of $73 \mu \mathrm{m}$, assuming a homogeneous glue layer and neglecting interface resistances. Importing this value into 


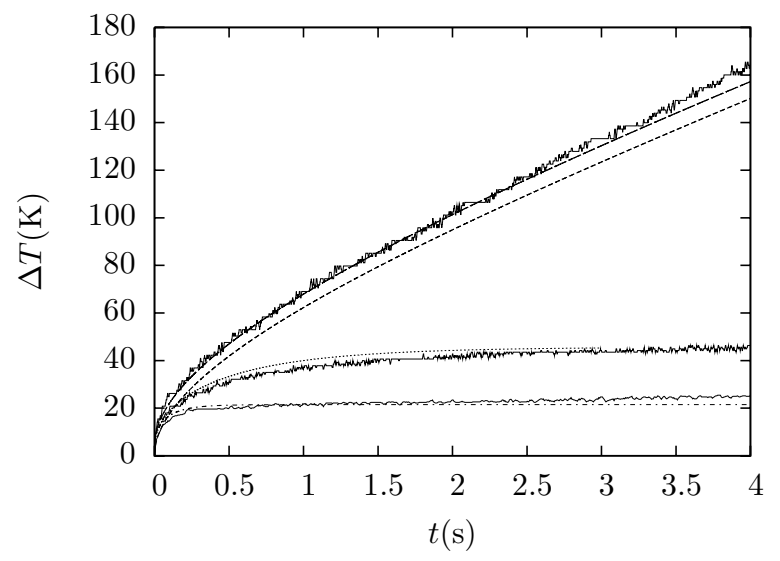

FIG. 3: Wire heating at long times for a $200 \mu \mathrm{m}$ wide-wire running a current of $5 \mathrm{~A}$. Solid lines give the experimental results for, from top to bottom, a chip laid on paper, a chip maintained on a copper block with a pressure of $2 \times 10^{3} \mathrm{~Pa}$ and a chip glued on the copper block. The other lines are theoretical predictions (see text).

the model, we compute the expected wire heating, shown in Fig. [II as a dashed-dotted line. It matches the time evolution very well.

We now turn to the measurements of the heating at early times. With a $200 \mu \mathrm{m}$ wide-wire, the early $2 D$ heating regime is barely visible, because the condition $W \ll e$ is not fulfilled. We thus use the $7 \mu \mathrm{m}$ wide-wire. The measurements were taken for a substrate laid on paper and are shown on Fig. 4. The prediction of the model with no free parameters, taking into account the $10 \mu$ s current rising time, is shown as solid line. For times between $100 \mu$ s and $5 \mathrm{~ms}$, we clearly observe a logarithmic increase of the temperature, as expected from the $2 D$ model, and the calculation agrees within $10 \%$ with the measurement. The dashed line is the result of the bare $2 D$ model of Eq. 2, including the temperature dependence of gold resistivity. $W_{\text {eff }}$ is adjusted to $0.15 W$, so that the bare $2 D$ model and the more complete model agree within $1 \%$ at times smaller than $5 \mathrm{~ms}$. At larger times, the model predicts a stronger heating due to the onset of the $1 D x$ regime, which is also visible in the experimental data. For times larger than $50 \mathrm{~ms}$, the model overestimates the temperature increase rate. This is very likely due to the finite length of the wire : indeed at $t=100 \mathrm{~ms}$, the heat has spread in the substrate over a typical distance $\sqrt{D t}=2.3 \mathrm{~mm}$ that is no longer negligible compared to the wire length $L=3 \mathrm{~mm}$. At longer times, the spread of the heat becomes two-dimensional in the plane of the substrate, which is more efficient for heat removal.

Because it is experimentally relevant, it is worth investigating briefly the effect of the finite wire length $L$ on the stationary temperature. As long as $l_{s} \ll L$, the equilibrium temperature is barely affected by the wire finite length. On the contrary, in the limit $l_{s} \gg L$, the model of an infinite wire fails. In this case, for times $t \gg L^{2} / D$, the wire temperature increases as $\Delta T^{2 D x y}(t) \simeq(\Phi L / 4 \pi e \lambda) \ln \left(D t / L^{2}\right)$ until the equilibrium temperature is reached. Equating the input power with the heat transferred to the heat sink, we find that the equilibrium temperature is about $T_{e q}^{2 D x y}=T_{e q}^{1 D x} \times$

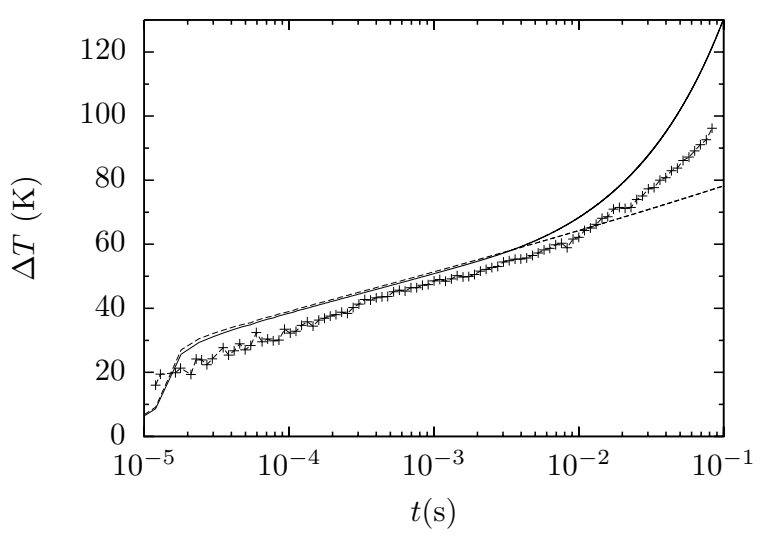

FIG. 4: Wire heating at long times for a $7 \mu \mathrm{m}$ wide-wire running a current of 1.7 A. Lines are predictions of the full model (solid) and the bare $2 D$ model of Eq. 2 (dashed). The crosses are experimental data.

$\left(L / \pi l_{s}\right) \ln \left(l_{s} / L\right)$, a value much smaller than $T_{e q}^{1 D x}$ (see section 2). Quantitative predictions are obtained using a twodimensional version of Eq. 5, setting $\omega$ to 0 and integrating over wavevectors in the $\mathrm{x}$ and $\mathrm{y}$ directions. In the experimental case considered in this paper, for a chip glued to the copper block and for our $3 \mathrm{~mm}$ long-wire (for which $l_{s}=0.96 L$ ), $T_{e q}$ is decreased by about $25 \%$ compared to its value for an infinite wire.

The data from Fig. 4 can additionally be used to place an upper limit on the thermal contact resistance $\sigma_{W}$ between the wire and the AlN substrate. In presence of a finite $\sigma_{W}$, the wire temperature increases by $\sigma_{W} \Phi / W$ on a time scale $\tau_{W}=\sigma_{W} C_{A u} h$, typically smaller than $1 \mu \mathrm{s}$, where $C_{A u}$ is the heat capacity of gold. This fast heating has been observed in Si-based atom chips [18]. Our data however are compatible with a vanishing $\sigma_{W}$, since they agree with our model which assumes $\sigma_{W}=0$. More precisely, within the precision of our model and measurements, we can confidently say that the excess heating due to a finite $\sigma_{W}$ cannot be more than $10 \mathrm{~K}$ in the first $100 \mu \mathrm{s}$, which yields the upper limit $\sigma_{W}=2.3 \times 10^{-8} \mathrm{Km}^{2} / \mathrm{W}$, corresponding to a conductance of $4.3 \times 10^{7} \mathrm{~W} /\left(\mathrm{Km}^{2}\right)$. This contact resistance is a factor 6.6 smaller than the values reported in [18].

\section{PRACTICAL CONSEQUENCES}

In order to minimize the wire temperature, it is of course desirable to minimize the thermal contact resistance $\sigma$ between the substrate and the reservoir. However, once a technology is chosen, so once $\sigma$ and $l_{c}$ are fixed, it is possible to use our model to optimize the chip design and to compute the maximal currents that can flow in each wire.

As we have shown from our measurements, in good operating conditions, a stationary state is expected to be reached within a few $100 \mathrm{~ms}$. This means that typical experiments on atoms will fall into the stationary regime; for example, an evaporation to BEC typically takes a few seconds in our set- 
up. Therefore the stationary temperature $\Delta T_{e q}$ is the relevant parameter to consider. In Fig. 5 a we plot $\Delta T_{e q}$ for different wire widths and substrate thicknesses. The scalings used are justified by dimensional analysis : since heat conduction inside the substrate is governed by linear equations, the equilibrium temperature is given by

$$
\Delta T_{e q}=\frac{\Phi}{\lambda} f\left(W / l_{c}, e / l_{c}\right)
$$

Note that the function $f$ does not depend on $c$, which only enters into account for the time scales of the transient regimes. One sees in Fig. 5] a that $\Delta T_{e q}$ is very well described by Eq.4. in its domain of validity $\left(W \ll e \ll l_{c}\right.$. Here $W_{0}=0.61 W$ has been obtained by fitting the calculation for $W=10^{-4} l_{c}$ and $5 \times 10^{-3}<e / l_{c}<0.3$. The failure of Eq. 4 for the lowest curve of Fig. 5] a at small $e / l_{c}$ is due to the fact that for these parameters $W$ is no longer small compare to $l_{s}$. Then, the $1 D x$ regime barely exists and only the initial $1 D z$ regime is present. Roughly speaking, in the case $e \ll l_{c}$, the temperature distribution has a 1D character, as can be seen in Fig. 5] b(B) which shows the temperature distribution inside the substrate for the parameters of Table 1. In this regime, it is favorable to increase $e$, to "deconfine" the energy spread. On the other hand, for $e \gg l_{c}$, the $1 D x$ regime of diffusion no longer exists : the steady state is realized directly after the $2 D$ regime when the energy reaches the substrate's lower surface. $\Delta T_{e q}$ is then of the order of $\ln (e / W) \Phi / \pi \lambda$, increasing with the substrate thickness (Fig. 5]a). These arguments predict an optimal substrate thickness of the order of $l_{c}$. This is confirmed by the calculations (Fig. 5a), which show that $\Delta T_{e q}$ reaches a minimum for the optimal substrate thickness $e^{*} \simeq 0.3 l_{c}$, which is about independent of $W$. In this optimal case, the three length scales $l_{s}, l_{c}$ and $e$ are almost equal. In fact, $e^{*}$ is the smallest value of $e$ that permits the suppression of the $1 D x$ regime and therefore the temperature distribution inside the substrate has a two-dimensional character (Fig. 5 b $(A)$ ). Note that the wire heating minimum is rather broad (Fig. 5]a) : even with a substrate thickness as low as $e^{*} / 10$, the heating is increased by only about $50 \%$.

The second important quantity we can derive from our model is the maximal current that can flow in the wires. Using Eq.7 and $\Phi=\rho I^{2} / W h$, we find that Eq. 8 is a self-consistent equation, whose solution is $\Delta T_{e q}=\rho_{0} I^{2} /(W h) f /(1-$ $\left.f l_{c} I^{2} / W I_{0}^{2}\right)$ where $I_{0}=\lambda \sqrt{\sigma h / \rho_{0} \alpha}$. When $I$ approaches the critical current $I_{c}=I_{0} \sqrt{W /\left(l_{c} f\right)}$ a divergence occurs, signature of an instability. In Fig. 6 we show the critical current $I_{c}$, where the dependence on $\sqrt{W}$ has been removed by dividing by $\sqrt{W / l_{c}}$. Note that this maximal current corresponds to an established stationary state. For situations where currents are only required for a time shorter than $t_{s}$, higher currents can be used.

\section{CONCLUSION}

To conclude, we have presented the first study on the thermal properties of AlN-based atom chips. The main result is that, as expected, the thermal behavior is more favorable than a)

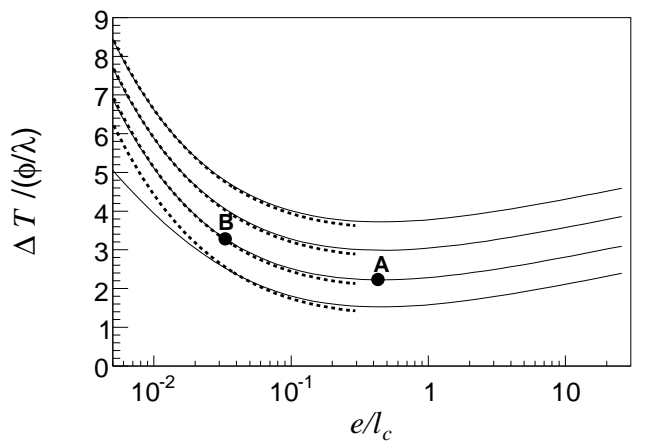

b)
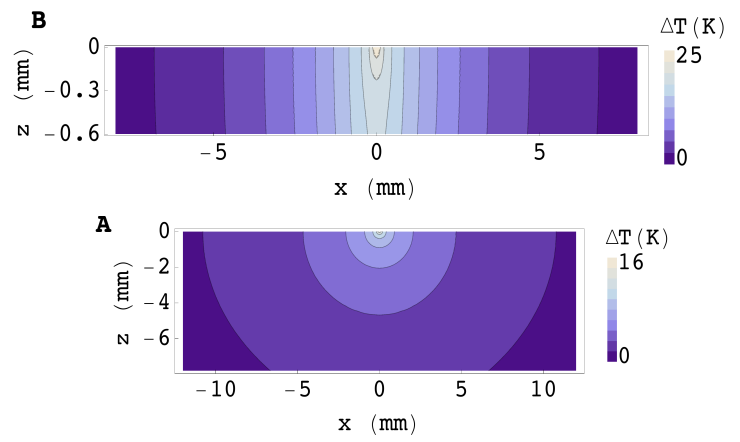

FIG. 5: Optimum substrate thickness. a) Equilibrium wire temperature versus substrate thickness for wire widths (from top to bottom) $W / l_{c}=10^{-4}, 10^{-3}, 1.1 \times 10^{-2}$, and 0.1 . The dashed lines correspond to Eq. 4 with $W_{\text {eff }}=0.61 W$. Heating is minimum for a substrate thickness of $0.3 l_{c}$. b) Stationary temperature distribution for the parameters of our chip glued on copper, for a substrate of thickness $e=0.6 \mathrm{~mm}$ (B) and for a substrate of optimal thickness $e^{*}=7.8 \mathrm{~mm}$ (A). The wire, $200 \mu \mathrm{m}$ wide, runs a current of $5 \mathrm{~A}$ and its temperature increase is $24.3 \mathrm{~K}$ in case $\mathrm{B}$ and $15.8 \mathrm{~K}$ in case $\mathrm{A}$.

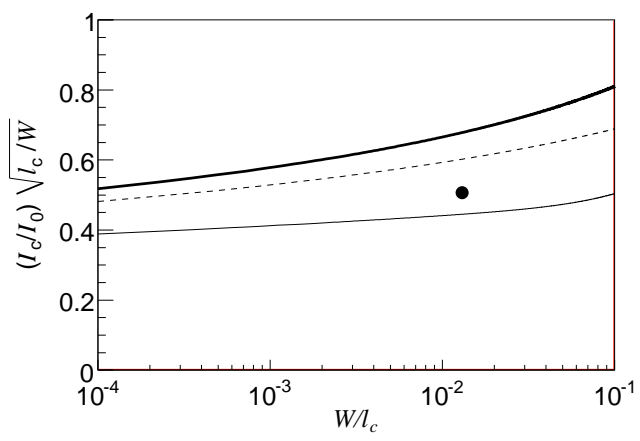

FIG. 6: Critical current divided by $\sqrt{W / l_{c}}$ and normalized to $I_{0}=$ $\lambda \sqrt{\sigma h / \rho_{0} \alpha}$, versus wire width for substrate thicknesses $e / l_{c}=0.5$ (thick line, optimal case), 10 (dashed line) and 0.01 (thin line). The point represents the case studied in this paper : a $200 \mu \mathrm{m}$ wide, $3 \mu \mathrm{m}$ high-wire on an AlN substrate of width $e=600 \mu \mathrm{m}$, for the parameters of Table 1 . In this case, $I_{c}=16 \mathrm{~A}$. 
with Si chips. More precisely, our measurements are compatible with the absence of thermal contact resistance between the wire and the substrate. The heating of the wire is entirely explained by heat diffusion inside the substrate and its absorption by the heat reservoir holding the chip. We have developped a model that accounts well for our experimental data for wires of different widths, for different couplings to the copper block and within the different heating regimes. The thermal coupling to the heat sink holding the chip is a crucial parameter : in particular we have shown that, when operating in vacuum, it is recommended to glue the chip to the heat sink. Finally, the model is used to derive pratical learnings : the optimum substrate thickness is computed as well as the maximum current that can be run into the wires in the stationary regime.

We have treated a simplified case, but, in practical situations, it is important to take other effects into account. First, as we already noticed, the finite length of the wire may reduce significantly the heating. The vicinity of wires may also affect the thermal behavior : several current-carrying wires separated by distances on the order or smaller than the stationary length $l_{s}$, will experience a stronger heating. In the temporal domain, the accumulation of heat over several experimental cycles will increase the heating if the dead time when currents are off is not long compared to $t_{s}$. Finally, heat conduction through the copper block and its cooling may affect the heating on long timescales. Active cooling of the copper block may be envisioned.

Different technologies may improve the thermal properties of atom chips. An expensive solution would be to use diamond substrates because diamond, although electrically insulating, has a much higher thermal conductivity than AIN. Another key point is the realization of a better thermal contact between the substrate and the heat sink. If the backside of the chip is in air, using a thermal grease seems highly desirable. Working in vacuum, a process leading to a thinner glue layer may be developped and/or a different glue may be used. One could also consider soldering, the difficulty being to avoid damaging the chip.

The Atom Optics group of Laboratoire Charles-Fabry is a member of the IFRAF Institute. This work was supportedd by the ANR grant ANR-08-BLAN-0165-03 and by EU under the grant IP-CT-015714.
[1] R. Folman et al., Adv. At. Mol. Opt. Phy. 48, 263 (2002), and ref. therein.

[2] H. Ott et al., Phys. Rev. Lett. 87, 230401 (2001).

[3] W. Hänsel, P. Hommelhoff, T. W. Hänsch, and J. Reichel, Nature 413, 498 (2001).

[4] J. Fortagh and C. Zimmermann, Rev. of Mod. Phys. 79, 235(55) (2007), and ref. therein.

[5] J. Esteve et al., Phys. Rev. Lett. 96, 130403 (2006).

[6] J.-B. Trebbia, J. Esteve, C. I. Westbrook, and I. Bouchoule, Phys. Rev. Lett. 97, 250403 (2006).

[7] A. H. van Amerongen et al., Phys. Rev. Lett. 100, 090402 (2008).

[8] S. Hofferberth et al., Nature Phys. 4, 489 (2008).

[9] in Atom Chips (J. Reichel and V. Vuletic, ADDRESS, In press), Chap. Fermions on atom chips.

[10] S. Wildermuth et al., Phys. Rev. A 69, 030901 (2004).

[11] J. Estève et al., Phys. Rev. A 70, 043629 (2004).

[12] S. Wildermuth et al., Nature 435, 440 (2005).

[13] Y. ju Lin, I. Teper, C. Chin, and V. Vuletić, Phys. Rev. Lett. 92, 050404 (2004).

[14] J. M. Obrecht et al., Phys. Rev. Lett. 98, 063201 (2007).

[15] C. Henkel, S. Pötting, and M. Wilkens, Appl. Phys. B 69, 379
(1999).

[16] D. Harber, J. McGuirk, J. Obrecht, and E. Cornell, J. Low Temp. Phys. 133, 229 (2003).

[17] T. Nirrengarten et al., Phys. Rev. Lett. 97, 200405 (2006)

[18] S. Groth et al., Appl. Phys. Lett. 85, 2980 (2004).

[19] W. Werdecker and F. Aldinger, IEEE Transactions on Components, Hybrids, and Manufacturing Technology 7, 399 (1984).

[20] B. Lev, Quantum Information and Computation 3, 450 (2003).

[21] Y. Hui, G.-Q. Yang, J. Wang, and M.-S. Zhan, Chinese Physics Letters 25, 3219 (2008).

[22] S. Du et al., Phys. Rev. A 70, 053606 (2004).

[23] in CRC Handbook of Chemistry and Physics (CRC Press, Boca Raton, Florida (USA), 2008).

[24] J. Dirker, Ph.D. thesis, University of Johannesburg, http://hdl.handle.net/10210/1739 2008.

[25] On distances much smaller than $\sqrt{D t}$, where $t$ is the typical timescale considered, the temperature distribution obeys the two-dimensional Laplace equation $\Delta T=0$. Using the complex variable $x+i z$ and a Schwartz-Christoffel conformal transformation, we find that $T \propto \mathcal{R e}(\operatorname{Arc} \operatorname{Cosh}(\mathrm{x}+\mathrm{iz}))$. The energy flux from the wire is $j(x)=-\lambda \partial T / \partial z)_{z=0}$. 